\title{
Desfechos clínicos de pacientes após uso de terapia fibrinolítica pré-hospitalar: revisão sistemática
}

Clinical outcomes of patients after using prehospital fibrinolytic therapy: a systematic review

Resultados clínicos de pacientes después del uso de terapia fibrinolítica prehospitalaria: revisión sistemática

Priscila Fernandes Meireles Câmara' ${ }^{1}$ io https://orcid.org/0000-0003-0433-7471

Marcos Antonio Ferreira Júnior² id https://orcid.org/0000-0002-9123-232X

Allyne Fortes Vitor ${ }^{1}$ io https://orcid.org/0000-0002-4672-2303

Viviane Euzébia Pereira Santos ${ }^{1}$ id https://orcid.org/0000-0001-8140-8320

Oleci Pereira Frota ${ }^{2}$ id https://orcid.org/0000-0003-3586-1313

Mayk Penze Cardoso² io https://orcid.org/0000-0003-2130-5738

Como citar:

Câmara PF, Ferreira Júnior MA, Vitor AF, Santos VE, Frota OP, Cardoso MP. Desfechos clínicos de pacientes após uso de terapia fibrinolítica pré-hospitalar: revisão sistemática. Acta Paul Enferm. 2020;33:eAPE20190094.

DOI

http://dx.doi.org/10.37689/actaape/2020AR00946

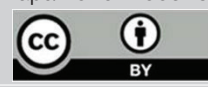

Descritores

Infarto do miocárdio; Terapia trombolítica; Fibrinolíticos; Serviços médicos de emergência

Keywords

Myocardial infarction; Thrombolytic therapy; Fibrinolytic agents; Emergency medical services

Descriptores

Infarto del miocardio: Terapia trombolítica; Fibrinolíticos; Servicios médicos de urgencia

\section{Submetido \\ 22 de Abril de 2019 \\ Aceito \\ 10 de Março de 2020}

Autor correspondente

Mayk Penze Cardoso

E-mail: maykcardoso@hotmail.com

\section{Resumo}

Objetivo: Comparar desfechos clínicos de óbito, reinfarto e Acidente Vascular Encefálico (AVE) em estudos primários que avaliaram o uso da Terapia Fibrinolítica (TF) em relação à Intervenção Coronariana Percutânea Primária (ICPP) para reperfusão miocárdica em pacientes com Infarto Agudo do Miocárdio com supradesnivelamento do segmento ST (IAMCST) no atendimento pré-hospitalar.

Método: Revisão sistemática de literatura com busca realizada nas bases de dados CINAHL, MEDLINE, PUBMED, Science Direct, SCOPUS e Web of Science no período de outubro a dezembro de 2017. Foram incluídos Ensaios Clínicos Randomizados, disponíveis na íntegra, em qualquer idioma, sem recorte temporal. A avaliação da elegibilidade foi realizada em duas etapas e aplicada a Escala de Jadad para avaliação metodológica dos estudos encontrados.

Resultados: Foram incluídos cinco Ensaios Clínicos Randomizados. A TF pré-hospitalar apresentou taxas de mortalidade em 30 dias após a intervenção semelhantes à ICPP, enquanto que em cinco anos foram encontrados valores menores para a TF. 0 tratamento instituído em um período menor que duas horas dos sintomas iniciais apresentou associação com a diminuição da mortalidade quando foi utilizada a TF. 0 reinfarto não-fatal, acidente vascular encefálico e a hemorragia intracraniana foram maiores quando utilizada a TF, enquanto que o choque cardiogênico apresentou menor frequência.

Conclusão: A TF foi mais eficaz no tratamento pré-hospitalar para a redução dos óbitos após cinco anos, entretanto, o reinfarto e o AVE ocorreram de forma semelhante na amostra analisada. 0 fator tempo reduziu os desfechos clínicos, principalmente quando a terapia implementada ocorreu em até duas horas após a ocorrência do IAMCST. Assim, apesar das intervenções terem apresentado desfechos semelhantes, entretanto, a TF pode representar um tratamento viável em locais onde a ICPP não pode ser alcançada em tempo hábil.

\section{Abstract}

Objective: To compare clinical outcomes of death, reinfarction, and stroke in primary studies assessing Fibrinolytic Therapy (FT) use in relation to Primary Percutaneous Coronary Intervention (PPCl) for myocardial reperfusion in patients with ST-Elevation Myocardial Infarction (STEMI) in prehospital care.

Method: A systematic literature review conducted in the CINAHL, MEDLINE, PUBMED, Science Direct, SCOPUS, and Web of Science databases from October to December 2017. Randomized Clinical Trials, available in full, in any language, without temporal clipping were included. The eligibility assessment was carried out in two stages and applied to the Jadad Scale for methodological assessment of the studies found.

Results: Five Randomized Clinical Trials were included. Prehospital FT presented mortality rates at 30 days after the intervention similar to PPCl, while in five years lower values were found for $\mathrm{FT}$. The treatment instituted 
in a period of less than two hours of the initial symptoms was associated with the decrease in mortality when FT was used. Non-fatal reinfarction, stroke and intracranial hemorrhage were higher when FT was used, while cardiogenic shock showed lower frequency.

Conclusion: FT was more effective in prehospital treatment to reduce deaths after five years, however, reinfarction and stroke occurred similarly in the sample analyzed. The time factor reduced clinical outcomes, especially when the implemented therapy occurred within two hours after the occurrence of STEMI. Thus, although the interventions presented similar outcomes. However, FT may represent a viable treatment in places where PPCl cannot be achieved in a timely manner.

\section{Resumen}

Objetivo: Comparar resultados clínicos de fallecimiento, reinfarto y accidente vascular encefálico (AVE) en estudios primarios que analizaron el uso de la terapia fibrinolítica (TF) respecto a la intervención coronaria percutánea primaria (ICPP) para reperfusión miocárdica en pacientes con infarto agudo de miocardio con supradesnivel del segmento ST (IAMCST) en la atención prehospitalaria.

Método: Revisión sistemática de literatura con búsqueda realizada en las bases de datos CINAHL, MEDLINE, PUBMED, Science Direct, SCOPUS y Web of Science en el período de octubre a diciembre de 2017. Se incluyeron ensayos clínicos aleatorizados, con texto completo disponible, en cualquier idioma, sin recorte temporal. El análisis de elegibilidad se realizó en dos etapas y se aplicó la escala de Jadad para una evaluación metodológica de los estudios encontrados.

Resultados: Se incluyeron cinco ensayos clínicos aleatorizados. La TF prehospitalaria presentó índices de mortalidad 30 días después de la intervención semejantes a la ICPP, mientras que en cinco años se encontraron valores menores en la TF. El tratamiento aplicado en un período menor a dos horas desde los síntomas iniciales presentó una relación con la reducción de la mortalidad cuando se utilizó la TF. Los reinfartos no fatales, los accidentes vasculares encefálicos y las hemorragias intracerebrales fueron mayores cuando se utilizó la TF, mientras que los choques cardiogénicos presentaron menor frecuencia.

Conclusión: La TF fue más eficaz en el tratamiento prehospitalario para reducir los fallecimientos después de cinco años, sin embargo, los reinfartos y los AVE ocurrieron de forma semejante en la muestra analizada. El factor tiempo redujo los resultados clínicos, principalmente cuando la terapia implementada ocurrió hasta dos horas después del episodio del IAMCST. De esta forma, a pesar de que las intervenciones presentaron resultados semejantes, la TF puede representar un tratamiento viable en lugares donde la ICPP no puede realizarse a tiempo.

\section{Introdução}

As afecçôes cardiovasculares, com destaque para as doenças coronarianas, representam a principal causa de morbimortalidade e de incapacidades no contexto mundial. ${ }^{(1)} \mathrm{Da}$ mesma forma, é responsável pela morte de cerca de $20 \%$ da população brasileira acima dos trinta anos de idade, quando o infarto agudo do miocárdio representa a maior parte desses casos. $(2,3)$ Em razão de grande parte ocorrer no ambiente extra hospitalar, os serviços de atendimento pré-hospitalar devem estar preparados para avaliaçáo, diagnóstico rápido e preciso dos casos de pacientes com Infarto Agudo do Miocárdio com supradesnivelamento do segmento ST (IAMCST), bem como para decisão sobre qual terapia de reperfusão será oportunamente instituída, quer seja a Intervenção Coronariana Percutânea Primária (ICPP) ou a Terapia Fibrinolítica (TF). Atrasos nessa avaliação diagnóstica e instituição terapêutica apresentam relaçôes diretas com maiores taxas de mortalidade. ${ }^{(4-6)}$

Sabe-se que a ICPP trata do método preferencial para reperfusão de quadros de IAMCST, entretanto, em razão dos poucos serviços que oferecem tal procedimento, a TF constitui por muitas vezes a única opção terapêutica, principalmente em países subdesenvolvidos ou em desenvolvimento.
Diretrizes atuais apontam para o uso da TF em casos de inexistência ou não acesso ao serviço de ICPP, entretanto, a TF deve ser entendida como um procedimento inicial que não descarta o uso da ICPP. ${ }^{(7)}$

Restriçóes logísticas e de recursos muitas vezes inviabilizam o acesso em tempo adequado à ICPP, ofertada de forma limitada em diversas regióes do mundo. ${ }^{(8)} \mathrm{O}$ aumento do tempo despendido na decisão para instituição terapêutica pode causar aumento da lesão miocárdica e consequentemente da morbimortalidade. ${ }^{(9,10)}$ Portanto, o uso adequado de qualquer forma de terapia para reperfundir a musculatura cardíaca constitui maior importância do que a escolha da terapia propriamente dita. Atrasos para o tratamento efetivo podem ser reduzidos por meio do uso da TF pré-hospitalar. ${ }^{(4-6)}$

A TF consiste na terapia mais facilmente acessível e sua administração pode ser iniciada de forma mais precoce que a ICPP. ${ }^{(4-6)} \mathrm{O}$ benefício relativo da ICPP sobre a TF é tempo-dependente e a administração no contexto pré-hospitalar ainda é discutida cientificamente. A terapia de reperfusão ideal tem estimulado controvérsias e despertado interesses por parte da comunidade científica. ${ }^{(11)}$

De forma a elucidar a relevância da TF como modalidade alternativa à ICPP para atendimento dos casos de IAMCST, este estudo objetivou com- 
parar os desfechos clínicos de óbito, reinfarto e Acidente Vascular Encefálico em estudos primários que avaliaram o uso da TF em relação à ICPP para reperfusão miocárdica em pacientes com IAMCST no atendimento pré-hospitalar.

\section{Métodos}

Trata de uma Revisão Sistemática (RS) de literatura, conduzida e relatada de acordo com as diretrizes do Preferred Reporting Items for Systematic reviews and Meta-Analyse (PRISMA). ${ }^{(12)}$

Foram incluídos apenas Ensaios Clínicos Randomizados (ECR), disponíveis na íntegra, em qualquer idioma, que abordaram o uso da TF no atendimento pré-hospitalar comparado ao uso da ICPP como tratamento de reperfusão miocárdica em pacientes com IAMCST. Foram excluídos os editoriais, cartas ao editor, resumos, opiniôes de especialistas, revisóes, resenhas, livros, capítulos de livros, teses, dissertaçóes, monografias e trabalhos de conclusão de curso que não estivessem disponíveis no formato de artigo científico publicados em revistas científicas nas bases de dados adotadas para busca.

A questão norteadora para alcance do objetivo proposto foi estruturada por meio da estratégia do acrônimo PICO, quando significaram: P: paciente com IAMCST atendido pelo serviço pré-hospitalar e tratado com terapia de reperfusão; I: Terapia Fibrinolítica; C: Intervenção Coronariana Percutânea Primária; O: óbito, reinfarto e Acidente Vascular Encefálico. ${ }^{(13)}$ Assim, chegou-se a seguinte questão norteadora: $\mathrm{O}$ uso da TF no atendimento pré-hospitalar comparado ao uso da ICPP para reperfusão do miocárdio em pacientes com IAMCST reduz os casos de óbito, reinfarto e Acidente Vascular Encefálico?

Foi elaborado um instrumento para coleta dos dados. A busca foi realizada de outubro a dezembro de 2017 nas seguintes bases de dados bibliográficas eletrônicas acessadas pelo proxy da Universidade Federal do Rio Grande do Norte: Cumulative Index to Nursisng and Allied Heath Literature (CINAHL); Medical Literature Analysis and Retrieval System On-line (MEDLINE); National Library of Medicine (PUBMED); Science Direct, SCOPUS e Web of Science.
Foram utilizados somente descritores controlados e seus respectivos Entry Terms identificados no Medical Subject Headings, conforme o quadro 1.

Para realização das buscas nas bases de dados, os descritores do quadro 1 foram combinados e siste-

Quadro 1. Descritores e respectivos entry terms do Medical Subject Headings utilizados nos cruzamentos para realização das buscas dos estudos

\begin{tabular}{|l|l|}
\hline Descritor controlado & Entry Terms \\
\hline $\begin{array}{l}\text { A. Myocardial } \\
\text { infarction }\end{array}$ & $\begin{array}{l}\text { infarction, myocardial; myocardial infarctions; heart attack; } \\
\text { myocardial infarct; infarct, myocardial. }\end{array}$ \\
\hline $\begin{array}{l}\text { B. Myocardial } \\
\text { reperfusion }\end{array}$ & $\begin{array}{l}\text { reperfusion, myocardial; myocardial reperfusions; coronary } \\
\text { reperfusions; reperfusion, coronary. }\end{array}$ \\
\hline C. Fibrinolytic agents & $\begin{array}{l}\text { thrombolytic agents; thrombolytic drugs; fibrinolytic drugs; } \\
\text { antithrombotic agents; antithrombic drugs. }\end{array}$ \\
\hline $\begin{array}{l}\text { D. Thrombolytic } \\
\text { therapy }\end{array}$ & $\begin{array}{l}\text { therapeutic thrombolyses; thrombolyses, therapeutic; therapy, } \\
\text { fibrinolytic; fibrinolytic therapies; therapy, thrombolytic; therapies, } \\
\text { thrombolytic. }\end{array}$ \\
\hline $\begin{array}{l}\text { E. Emergency medical } \\
\text { services }\end{array}$ & emergency services, medical; medical emergency service. \\
\hline
\end{tabular}

matizados em dois grandes cruzamentos, a saber: \#1 $\mathrm{A}$ and $\mathrm{B}$ and $\mathrm{C}$ and $\mathrm{E} \mathrm{e} \# 2 \mathrm{~A}$ and $\mathrm{B}$ and $\mathrm{D}$ and $\mathrm{E}$.

Após o processo de busca foi realizada a avaliação da elegibilidade dos artigos dos estudos em duas etapas. A primeira por meio de uma triagem pela leitura dos títulos e resumos realizada por dupla de revisores independentes. Quando dúvida, os artigos foram selecionados para a próxima fase. A segunda etapa foi a avaliação da elegibilidade pela leitura na íntegra do manuscrito. Foi realizada como forma de garantir a inclusão do número máximo de estudos, a busca nas referências dos artigos previamente selecionados nessa fase, bem como na literatura cinzenta sobre o tema, sem inclusão de novos trabalhos que atendessem os critérios pré-estabelecidos.

Os dados foram extraídos e organizados com o uso das fichas clínicas e organizados em quadro e tabelas. Do total de 5.207 artigos da amostra inicial, cinco foram selecionados para composição da amostra final (Figura 1).

Foi utilizada a Escala de Jadad para avaliação da qualidade metodológica dos ECR e como forma de minimizar as possibilidades de vieses, composta por três itens com respostas dicotômicas e dois subitens que avaliam a presença e adequação da randomização e duplo cegamento, além da descrição das perdas e exclusóes. ${ }^{(13)} \mathrm{O}$ valor máximo a ser obtido é de cinco pontos e o estudo foi considerado de qua- 


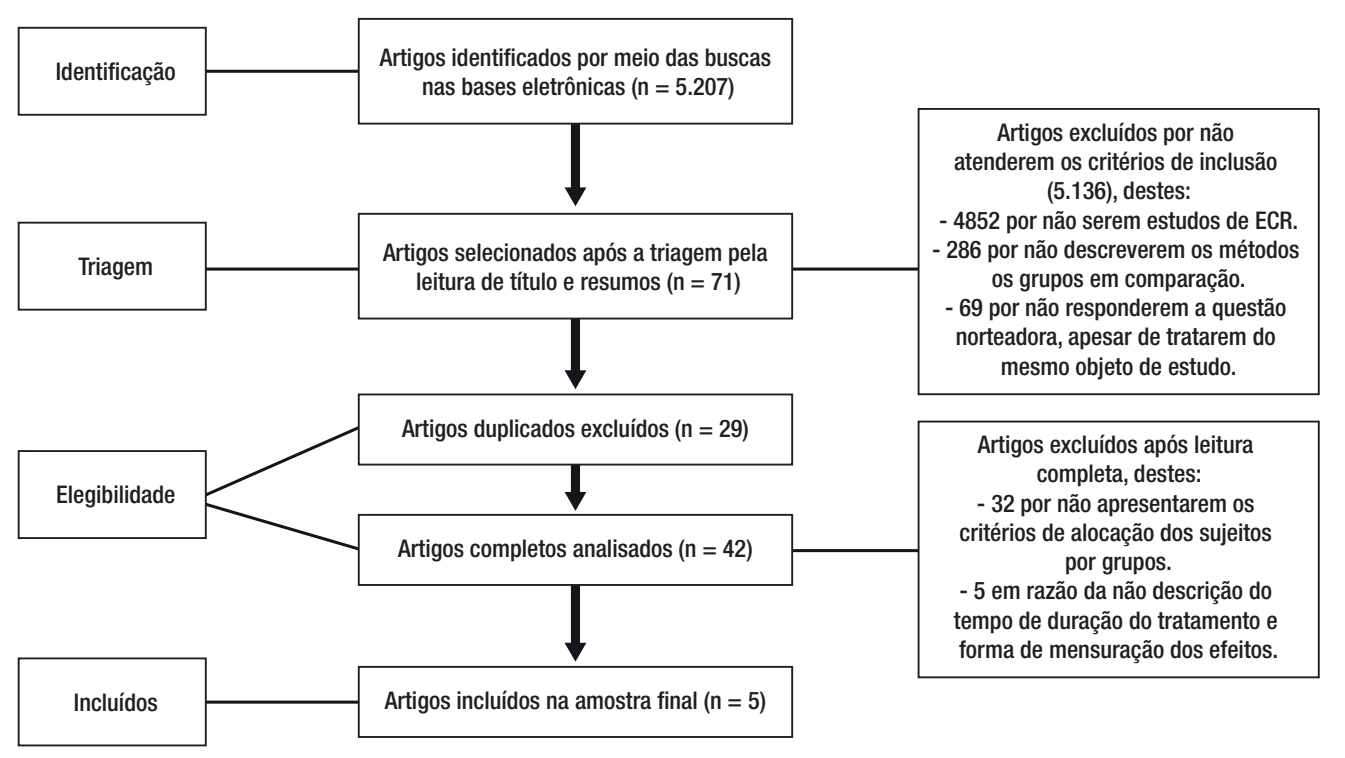

Figura 1. Fluxograma do processo de seleção dos artigos da amostra final

lidade metodológica a partir de três pontos. Assim, todos os estudos dessa revisão obtiveram pontuação três e foram considerados de boa qualidade metodológica.

\section{Resultados}

Dos cinco artigos incluídos, um é referente ao estudo Strategic Reperfusion Early after Myocardial Infarction (STREAM) realizado na Bélgica com 30 dias de seguimento após o tratamento e quatro referentes ao estudo Comparison of primary Angioplasty and Prehospital fibrinolysis In acute Myocardial infarction (CAPTIM), realizados na França com 30 dias de seguimento após o tratamento, exceto um com 5 anos de acompanhamento. ${ }^{(14-18)}$ Dos estudos CAPTIM, um foi realizado com acompanhamento a curto prazo, outro a longo prazo e dois com populaçóes específicas por características clínicas ou temporal. ${ }^{(15-18)}$

O quadro 2 apresenta as características metodológicas dos artigos, com os respectivos objetivos, principais resultados encontrados e conclusão obtida. O estudo mais recente foi o STREAM que fez uso do Tenecteplase, enquanto que no CAPTIM foi realizada a administração do Alteplase.
A tabela 1 apresenta os desfechos clínicos óbito, reinfarto e Acidente Vascular Encefálico (AVE) que foram considerados por quatro dos ECR no período de 30 dias após a terapêutica. Observou-se que o óbito foi semelhante entre os grupos em um ano, mas em cinco anos o número de mortes foi menor nos pacientes submetidos a TF, principalmente quando tratados nas primeiras duas horas.

Quando os pacientes foram submetidos a terapia até duas horas do início dos sintomas, o grupo TF apresentou menor número de óbitos, mas quando tratados após duas horas do início dos sintomas apresentaram mortalidade em cinco anos semelhantes, independente do tratamento.

Como dados complementares encontrados por esse estudo, os principais efeitos adversos após utilização da terapia de reperfusão adotada pelos ECR analisados foram agrupados e descritos na tabela $2 \mathrm{a}$ seguir. Tais informaçôes permitem complementar as implicações da adoção das modalidades comparadas por modalidade de tratamento. Dentre esses efeitos adversos, as hemorragias foram classificadas de formas distintas entre os estudos, quando a hemorragia intracraniana apresentou maior incidência no grupo TF pré-hospitalar, enquanto que com o choque cardiogênico ocorreu em maior número na ICPP. 
Quadro 2. Características dos ensaios clínicos randomizados e qualidade metodológica dos estudos incluídos na amostra final $(\mathrm{n}=5)$

\begin{tabular}{|c|c|c|c|c|c|c|c|c|}
\hline Artigo & Intervenções & $\begin{array}{l}\text { Amostra/ } \\
\text { participantes } \\
\text { por braço }\end{array}$ & $\begin{array}{l}\text { Terapia } \\
\text { fibrinolítica } \\
\text { utilizada }\end{array}$ & $\begin{array}{l}\text { Tempo entre } \\
\text { início dos } \\
\text { sintomas e } \\
\text { terapêutica }\end{array}$ & \begin{tabular}{|l} 
Escala \\
de \\
Jadad
\end{tabular} & Objetivo & Principais resultados & Conclusão \\
\hline$A 1^{(14)}$ & $\begin{array}{l}\text { TF pré- } \\
\text { hospitalar e } \\
\text { ICPP }\end{array}$ & $\begin{array}{l}\text { Amostra: } 1892 \\
\text { TF pré-hosp: } \\
944 \\
\text { ICPP: } 948\end{array}$ & Tenecteplase & $<3$ horas & 3 & $\begin{array}{l}\text { Verificar se a TF } \\
\text { pré-hospitalar é } \\
\text { mais efetiva em } \\
\text { comparação a ICPP } \\
\text { em pacientes com } \\
\text { sintomas precoces. }\end{array}$ & $\begin{array}{l}\text { Os desfechos de morte, choque, ICC ou reinfarto } \\
\text { em } 30 \text { dias foram discretamente menores } \\
\text { nos que receberam TF pré-hospitalar (12,4\%) } \\
\text { quando comparados aos que usaram a ICPP } \\
\text { (14,3\%). Foram constatadas mais hemorragias } \\
\text { intracranianas no grupo TF pré-hospitalar do } \\
\text { que no grupo ICPP e as demais hemorragias } \\
\text { ocorreram sem diferença entre os grupos. }\end{array}$ & $\begin{array}{l}\text { A TF pré-hospitalar resultou em } \\
\text { reperfusão efetiva em pacientes } \\
\text { com IAMCST que não puderam } \\
\text { receber a ICP primária dentro } \\
\text { de uma hora após o primeiro } \\
\text { contato. A TF pré-hospitalar } \\
\text { apresentou associação a um } \\
\text { risco um pouco maior de } \\
\text { sangramento intracraniano. }\end{array}$ \\
\hline$A 2^{(15)}$ & $\begin{array}{l}\text { TF pré- } \\
\text { hospitalar, ICP } \\
\text { de resgate e } \\
\text { ICPP }\end{array}$ & $\begin{array}{l}\text { Amostra: } 840 \\
\text { TF pré-hosp: } \\
419 \\
\text { ICPP: } 421\end{array}$ & Alteplase & $\begin{array}{l}\geq 30 \text { min. } e \\
<6 \text { horas. }\end{array}$ & 3 & $\begin{array}{l}\text { Verificar se a ICPP } \\
\text { é superior a TF pré- } \\
\text { hospitalar seguida } \\
\text { de ICP de resgate. }\end{array}$ & $\begin{array}{l}\text { A taxa dos desfechos primários foi de } 8,2 \% \text { no } \\
\text { grupo TF pré-hospitalar e } 6,2 \% \text { no grupo da } \\
\text { ICPP. A ICP de resgate foi realizada em } 26 \% \\
\text { dos pacientes que utilizaram TF pré-hospitalar. } \\
\text { Ocorreram } 16 \text { óbitos entre os pacientes } \\
\text { receberam TF pré-hospitalar e } 20 \text { entre os que } \\
\text { receberam ICPP. }\end{array}$ & $\begin{array}{l}\text { A ICPP não apresentou melhores } \\
\text { resultados dos desfechos } \\
\text { morte, reinfarto e AVE quando } \\
\text { comparada a TF pré-hospitalar } \\
\text { com transferência para uma } \\
\text { unidade de intervenção para } \\
\text { possível ICP de resgate. }\end{array}$ \\
\hline$A 3^{(16)}$ & $\begin{array}{l}\text { TF pré- } \\
\text { hospitalar, ICP } \\
\text { de resgate e } \\
\text { ICPP }\end{array}$ & $\begin{array}{l}\text { Amostra: } 840^{*} \\
\text { D: } 103 \\
\text { TF pré-hosp: } \\
46 \\
\text { ICPP: } 57 \\
\text { ND: } 731 \\
\text { TF pré-hosp: } \\
370 \\
\text { ICPP: } 361 \\
\end{array}$ & Alteplase & $\begin{array}{l}\geq 30 \text { min. } e \\
<6 \text { horas. }\end{array}$ & 3 & $\begin{array}{l}\text { Avaliar o impacto } \\
\text { do status diabético } \\
\text { nos benefícios } \\
\text { relativos das } \\
\text { estratégias de } \\
\text { reperfusão. }\end{array}$ & $\begin{array}{l}\text { Os diabéticos apresentaram um risco mais } \\
\text { alto, com uma taxa maior dos desfechos morte, } \\
\text { reinfarto e AVE (14,6 vs. 5,6\%) e uma alta } \\
\text { mortalidade }(8,7 \text { vs. 3,1\%) relacionados ao uso } \\
\text { da TF hospitalar em relação à ICPP, quando } \\
\text { comparados aos não diabéticos. }\end{array}$ & $\begin{array}{l}\text { Pacientes diabéticos atendidos } \\
\text { dentro de até } 6 \mathrm{~h} \text { após um } \\
\text { IAMCST podem ser beneficiado } \\
\text { pela ICPP. }\end{array}$ \\
\hline$A 4^{(17)}$ & $\begin{array}{l}\text { TF pré- } \\
\text { hospitalar, ICP } \\
\text { de resgate e } \\
\text { ICPP }\end{array}$ & $\begin{array}{l}\text { Amostra: } 840 \\
\text { TF pré-hosp: } \\
419 \\
\text { ICPP: } 421\end{array}$ & Alteplase & $\begin{array}{l}\geq 30 \text { min. e } \\
<6 \text { horas. }\end{array}$ & 3 & $\begin{array}{l}\text { Investigar a } \\
\text { mortalidade a longo } \\
\text { prazo (5 anos) dos } \\
\text { pacientes incluídos } \\
\text { no estudo. }\end{array}$ & $\begin{array}{l}\text { A mortalidade por todas as causas aos } 5 \text { anos } \\
\text { foi de } 9,7 \% \text { no grupo de TF pré-hospitalar, } \\
\text { comparada a } 12,6 \% \text { no grupo ICPP. Para } \\
\text { os pacientes atendidos em duas horas, a } \\
\text { mortalidade foi de } 5,8 \% \text { no grupo TF pré- } \\
\text { hospitalar, comparada com } 11,1 \% \text { no grupo ICPP. } \\
\text { Nos atendidos após duas horas os valores foram } \\
\text { de } 14,5 \text { e } 14,4 \% \text {, respectivamente. }\end{array}$ & $\begin{array}{l}\text { Para os pacientes tratados } \\
\text { dentro de até duas horas após } \\
\text { início dos sintomas do IAMCST, } \\
\text { a mortalidade em cinco anos fo } \\
\text { menor com naqueles atendidos } \\
\text { com uso da TF pré-hospitalar de } \\
\text { forma geral. }\end{array}$ \\
\hline$A 5^{(18)}$ & \begin{tabular}{|l} 
TF pré- \\
hospitalar, ICP \\
de resgate e \\
ICPP
\end{tabular} & $\begin{array}{l}\text { Amostra: } 840 \\
\text { TF pré-hosp: } \\
419 \\
\text { ICPP: } 421 \\
<2 \text { h: } 460 \\
\geq 2 \text { h: } 374\end{array}$ & Alteplase & $\begin{array}{l}\geq 30 \text { min. } e \\
<6 \text { horas. }\end{array}$ & 3 & $\begin{array}{l}\text { Analisar o efeito } \\
\text { do tratamento } \\
\text { instituído com TF } \\
\text { pré-hospitalar ou } \\
\text { ICPP em razão do } \\
\text { tempo decorrido } \\
\text { desde o início dos } \\
\text { sintomas. }\end{array}$ & $\begin{array}{l}\text { Pacientes tratados até duas horas do início dos } \\
\text { sintomas apresentaram mortalidade menor } \\
\text { quando tratados com TF fibrinolítica comparados } \\
\text { aos que receberam a ICPP até os } 30 \text { dias após } \\
\text { o evento. Os tratados após duas horas não } \\
\text { apresentaram diferenças para os desfechos } \\
\text { morte, reinfarto ou AVE por modalidade } \\
\text { terapêutica. }\end{array}$ & $\begin{array}{l}\text { A TF pré-hospitalar pode ser } \\
\text { preferivel em relação a ICPP } \\
\text { para pacientes tratados nas } \\
\text { duas primeiras horas após o } \\
\text { inicío dos sintomas. }\end{array}$ \\
\hline
\end{tabular}

*0 quadro clínico de seis pacientes era desconhecido em relação ao diabetes; TF - Terapia Fibrinolítica; ICP - Intervenção Coronariana Percutânea; ICPP - Intervenção Coronariana Percutânea Primária; D - Diabéticos; ND Não diabéticos; ICC - Insuficiência Cardíaca Congestiva; IAMCST - Infarto Agudo do Miocárdio com supradesnivelamento do segmento ST; AVE - Acidente Vascular Encefálico

Tabela 1. Desfechos primários e mediana do tempo entre 0 aparecimento dos sintomas e instituição terapêutica dos estudos incluídos na amostra final

\begin{tabular}{|c|c|c|c|c|c|c|c|c|c|c|c|c|c|c|}
\hline & \multirow{2}{*}{\multicolumn{4}{|c|}{ TF pré-hospitalar }} & \multicolumn{4}{|c|}{ Reinfarto } & \multicolumn{4}{|c|}{ AVE } & \multicolumn{2}{|c|}{$\begin{array}{l}\text { Mediana tempo para } \\
\text { tratamento (min.) }\end{array}$} \\
\hline \multirow[b]{2}{*}{$\mathrm{A} 1^{(14)}$} & & & & & & \multicolumn{2}{|c|}{ ICPP } & \multicolumn{2}{|c|}{ TF pré-hospitalar } & \multicolumn{2}{|c|}{ ICPP } & \multirow{2}{*}{$\begin{array}{c}\begin{array}{c}\text { TF pré- } \\
\text { hospitalar }\end{array} \\
\begin{array}{c}100 \\
(75-143)\end{array}\end{array}$} & \multirow{2}{*}{$\begin{array}{c}\text { ICPP } \\
178 \\
(135-230)\end{array}$} \\
\hline & $\begin{array}{l}\text { Qualquer causa } \\
43 / 939(4.6 \%)\end{array}$ & $\begin{array}{l}\text { Cardiovascular } \\
31 / 939(3.3 \%)\end{array}$ & $\begin{array}{l}\text { Qualquer causa } \\
42 / 946(4.4 \%)\end{array}$ & $\begin{array}{l}\text { Cardiovascular } \\
32 / 946(3.4 \%)\end{array}$ & \multicolumn{2}{|c|}{$\begin{array}{r}23 / 938 \\
(2,5 \%)\end{array}$} & \multicolumn{2}{|c|}{$\begin{array}{l}21 / 944 \\
(2,2 \%)\end{array}$} & \multicolumn{2}{|c|}{$\begin{array}{l}15 / 939 \\
(1,6 \%)\end{array}$} & \multicolumn{2}{|c|}{$5 / 946(0,5 \%)$} & & \\
\hline$A 2^{(15)}$ & \multicolumn{2}{|c|}{$\begin{array}{l}16 / 419 \\
(3,8 \%)\end{array}$} & \multicolumn{2}{|c|}{$\begin{array}{l}20 / 421 \\
(4,8 \%)\end{array}$} & \multicolumn{2}{|c|}{$\begin{array}{l}15 / 419 \\
(3,7 \%)\end{array}$} & \multicolumn{2}{|c|}{$\begin{array}{l}7 / 421 \\
(1,7 \%)\end{array}$} & \multicolumn{2}{|c|}{$\begin{array}{l}4 / 419 \\
(1,0 \%)\end{array}$} & \multicolumn{2}{|c|}{00} & $\begin{array}{c}130 \\
(95-180)\end{array}$ & $\begin{array}{c}190 \\
(149-255)\end{array}$ \\
\hline$A 3^{(16)}$ & $\begin{array}{c}D \\
6 / 46 \\
(13 \%)\end{array}$ & $\begin{array}{c}\text { ND } \\
9 / 370 \\
(2,4 \%)\end{array}$ & $\begin{array}{c}D \\
3 / 57 \\
(5,3 \%)\end{array}$ & $\begin{array}{c}\text { ND } \\
14 / 361 \\
(3,9 \%)\end{array}$ & $\begin{array}{c}D \\
3 / 40 \\
(7,5 \%)\end{array}$ & $\begin{array}{c}N D \\
12 / 361 \\
(3,3 \%)\end{array}$ & $\begin{array}{c}D \\
3 / 55 \\
(5,5 \%)\end{array}$ & $\begin{array}{c}\mathrm{ND} \\
4 / 346 \\
(1,2 \%)\end{array}$ & $\begin{array}{c}D \\
1 / 46 \\
(2,5 \%)\end{array}$ & $\begin{array}{c}\text { ND } \\
3 / 370 \\
(0,9 \%)\end{array}$ & $\begin{array}{c}D \\
00 \\
-\end{array}$ & $\begin{array}{c}\text { ND } \\
00 \\
-\end{array}$ & $\begin{array}{l}\text { D: } 160(1 \\
\text { ND: } 155\end{array}$ & $\begin{array}{l}7.5-225) \\
115-220)\end{array}$ \\
\hline \multirow[t]{2}{*}{$\mathrm{A} 4^{(17)}$} & $\begin{array}{c}1 \text { ano } \\
23 / 419 \\
(5,5 \%)\end{array}$ & $\begin{array}{c}5 \text { anos } \\
40 / 419(9,7 \%)\end{array}$ & $\begin{array}{c}1 \text { ano } \\
30 / 421 \\
(7,1 \%)\end{array}$ & $\begin{array}{c}5 \text { anos } \\
52 / 421 \\
(12,6 \%)\end{array}$ & \multirow{2}{*}{\multicolumn{2}{|c|}{$\mathrm{N} / \mathrm{A}$}} & \multirow{2}{*}{\multicolumn{2}{|c|}{$\mathrm{N} / \mathrm{A}$}} & \multirow{2}{*}{\multicolumn{2}{|c|}{$\mathrm{N} / \mathrm{A}$}} & \multirow{2}{*}{\multicolumn{2}{|c|}{$\mathrm{N} / \mathrm{A}$}} & \multirow{2}{*}{\multicolumn{2}{|c|}{$\begin{array}{c}130 \\
(95-180)\end{array}$}} \\
\hline & $\begin{array}{l}5 \text { anos } \\
<2 h^{1} \\
(5,8 \%)\end{array}$ & $\begin{array}{c}5 \text { anos } \\
>2 h^{2} \\
(14,5 \%)\end{array}$ & $\begin{aligned} & 5 \text { anos } \\
< & 2 h^{1}(11,1 \%)\end{aligned}$ & $\begin{array}{c}5 \text { anos } \\
>2 h^{2} \\
(14,4 \%)\end{array}$ & & & & & & & & & & \\
\hline$A 5^{(18)}$ & $\begin{array}{l}<2 h^{1} \\
5 / 460 \\
(2,2 \%)\end{array}$ & $\begin{array}{c}>2 h^{2} \\
11 / 374 \\
(5,9 \%)\end{array}$ & $\begin{array}{c}<2 \mathrm{~h}^{1} \\
13 / 460 \\
(5,7 \%)\end{array}$ & $\begin{array}{l}>2 h^{2} \\
7 / 374 \\
(3,7 \%)\end{array}$ & $\begin{array}{c}<2 h^{1} \\
9 / 460 \\
(4,0 \%)\end{array}$ & $\begin{array}{l}>2 h^{2} \\
6 / 374 \\
(3,4 \%)\end{array}$ & $\begin{array}{l}<2 h^{1} \\
3 / 460 \\
(1,4 \%)\end{array}$ & $\begin{array}{l}>2 h^{2} \\
4 / 374 \\
(2,2 \%)\end{array}$ & $\begin{array}{l}<2 h^{1} \\
3 / 460 \\
(1,3 \%)\end{array}$ & $\begin{array}{c}>2 h^{2} \\
1 / 374 \\
(0,6 \%)\end{array}$ & $\begin{array}{c}<2 h^{1} \\
00 \\
-\end{array}$ & $\begin{array}{c}>2 h^{2} \\
00 \\
-\end{array}$ & $\begin{array}{l}<2 \mathrm{~h} \\
(40- \\
\geq 2 \mathrm{~h} \\
(120-\end{array}$ & $\begin{array}{l}: 120 \\
260) \\
: 225 \\
1275)\end{array}$ \\
\hline
\end{tabular}

AVE - Acidente Vascular Encefálico; TF - Terapia Fibrinolítica; ICP - Intervenção Coronariana Percutânea; ICPP - Intervenção Coronariana Percutânea Primária; D - Diabéticos; ND - Não diabéticos; 1 - Pacientes tratadoS dentro de duas horas após início dos sintomas; 2 - Pacientes tratados de 2 a 6 horas após o início dos sintomas; N/A - Não se aplica 
Tabela 2. Principais eventos adversos após instituição da terapia de reperfusão dos estudos incluídos na amostra final $(\mathrm{n}=5)$

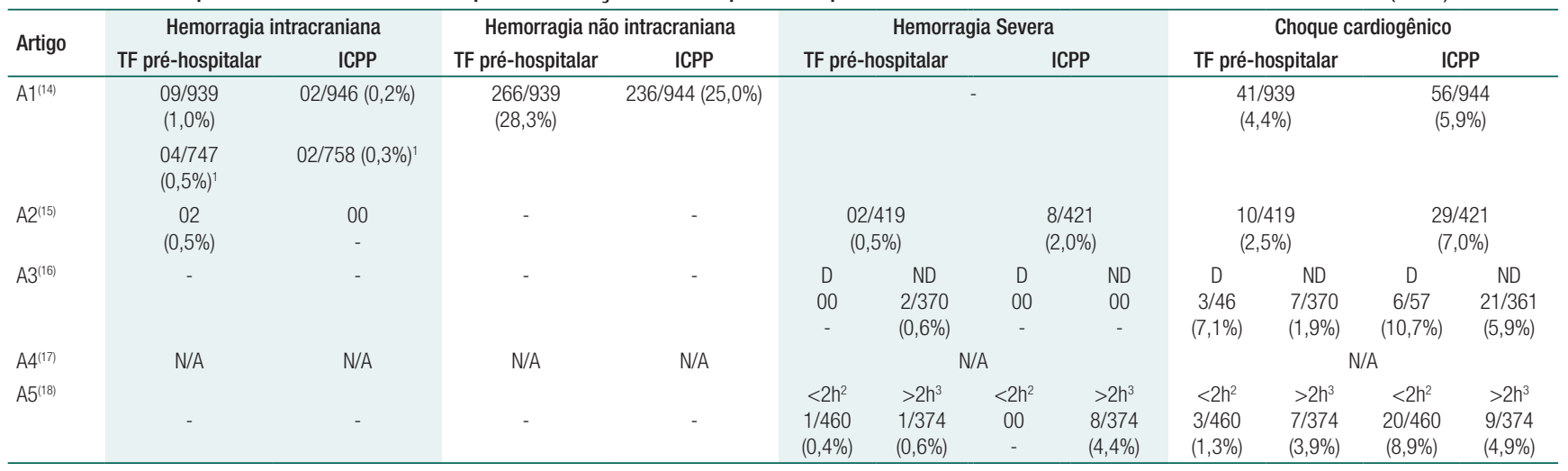

TF - Terapia Fibrinolitica; ICP - Intervenção Coronariana Percutânea; ICPP - Intervenção Coronariana Percutânea Primária; D - Diabéticos; ND - Não diabéticos; 1 - Reajuste da dose do fibrinolíticos para metade nos pacientes com mais de 75 anos; 2 - Pacientes tratados dentro de duas horas após início dos sintomas; 3 - Pacientes tratados de 2 a 6 horas após 0 início dos sintomas

\section{Discussão}

Diversos estudos foram encontrados sobre as modalidades terapêuticas de reperfusão do miocárdio a ser adotada em casos de IAMCST, entretanto, apenas dois grandes estudos, conduzidos por meio de ECR foram conduzidos de forma controlada para avaliação das modalidades de TF pré-hospitalar comparada a ICCP, razão pela qual apenas cinco publicações foram incluídas nessa revisão.

Como forma de organizar didaticamente a sessão de discussão, esta foi subdividida por tópicos que abordam a discussão dos resultados relacionados a conduta terapêutica de reperfusão, a escolha pela TF, o tempo para instituição da terapêutica de reperfusão, a intervenção coronariana pós TF pré-hospitalar, os desfechos primários e os eventos adversos após o uso da terapia de reperfusão.

\section{Conduta terapêutica de reperfusão miocárdica}

Em pacientes com IAMCST, a ICPP constitui a estratégia de reperfusão padrão recomendada, quando realizada preferencialmente nos primeiros 90 minutos após contato médico. ${ }^{(1)}$ Contudo, a maioria dos pacientes não possui acesso à ICPP, até mesmo em razão da estrutura de serviços de saúde específicos disponibilizada, logo esta estratégia constitui grande desafio para muitos países. ${ }^{(19)}$

Muitas regiôes dos Estados Unidos da América possuem acesso à ICPP, enquanto na Europa o uso do fibrinolítico pré-hospitalar é a estratégia que mais cresce pela sua possibilidade de acesso em tempo adequado. No Brasil os serviços encontram-se concentrados nos grandes centros, muitas vezes em quantidade insuficiente para atendimento a toda população e distantes das áreas de cobertura. (4) Entretanto, é consenso que deve ser dado ênfase na administraçáo terapêutica em tempo oportuno da terapia de reperfusão ao invés do modo de tratamento propriamente dito. ${ }^{(20)}$

A TF apresentou desfechos semelhantes à ICPP ou ainda superiores quando administrada até duas horas após o início do sintomas. ${ }^{(18)}$ Uma grande vantagem da TF é a possibilidade de diminuição do tempo para administração por meio dos serviços pré-hospitalares, principalmente em países em que há poucos centros capazes de realizar a ICPP, como é o caso do Brasil. ${ }^{(4,21,22)}$

O estudo STREAM relatou efetividade da TF pré-hospitalar quando comparada à ICPP, já o estudo CAPTIM afirma não ter encontrado superioridade da ICPP. ${ }^{(14)} \mathrm{A}$ partir do adiantamento do CAPTIM, percebeu-se mortalidade semelhante no período de 30 dias após o evento inicial, mas para pacientes tratados dentro das duas primeiras horas do início dos sintomas a mortalidade em cinco anos foi menor com TF pré-hospitalar. ${ }^{(15-17)} \mathrm{A}$ instituição precoce da terapia de reperfusão significa menor tempo de isquemia miocárdica, com lesôes menos extensas, danos sistêmicos de menor repercussão e probabilidades acumuladas de sobrevida maiores, o que confirma que a efetividade da modalidade adotada será tempo-dependente para redução dos desfechos negativos e melhores prognósticos. ${ }^{(14-18)}$ 


\section{Escolha pela droga fibrinolítica}

A TF pode ser administrada no ambiente pré-hospitalar ou hospitalar, quando é mais eficaz se administrada nos primeiros minutos após o início dos sintomas do IAMCST. ${ }^{(23)}$

O alteplase consiste em um fibrinolítico com alta eficácia e segurança com meia-vida curta, o que requer uma administração complexa. $\mathrm{O}$ tenecteplase é um fibrinolítico de terceira geração com meia-vida mais longa e especificidade mais elevada à fibrina, logo pode ser administrado em bolus único, o que torna a administração pré-hospitalar mais segurança e ágil, além de facilitar a terapia de reperfusão no atendimento pré-hospitalar. ${ }^{(24-26)}$

O estudo CAPTIM utilizou o alteplase e identificou que o tempo para administrar o medicamento retardou a terapia de reperfusão medicamentosa. ${ }^{(15)}$ Assim, o uso do tenecteplase facilita a administração e otimiza o tempo.

\section{Tempo para instituição da terapêutica de reperfusão}

O objetivo absoluto do tratamento do paciente com IAMCST é conseguir a reperfusão o mais precoce possível, uma vez que esses tratamentos são tempo-dependentes. ${ }^{(20)}$ Os estudos ${ }^{(14-18)}$ evidenciaram que o tempo despendido entre o início dos sintomas e a instituição da terapia de reperfusão foi menor quando adotada a TF pré-hospitalar em comparação à ICPP. Percebeu-se ainda uma diminuição na mediana de tempo quando o estudo apresentou a população com sintomatologia até duas horas.

\section{Intervenção coronariana pós TF pré-hospitalar}

Diretrizes norte-americanas e europeias indicam que os pacientes submetidos a TF pré-hospitalar devem ser encaminhados para realização de angiografia nas próximas seis a 24 horas. ${ }^{(4,27)}$ Ensaios clínicos com pacientes que foram tratados com fibrinolítico, demonstraram que a angiografia realizada rotineiramente após a reperfusão medicamentosa com ICP, se necessária, está associada a um número significativamente menor de complicaçóes isquêmicas com reduçáo das taxas de reinfarto. ${ }^{(28-30)}$ Nos estudos CAPTIM e STREAM, após a TF pré-hospitalar os pacientes foram encaminhados para centros capazes de realizar angiografia. Da população estudada, $70,4 \%$ e $80,4 \%$ dos pacientes foram submetidos à ICP, respectivamente, o que conferiu elevado número de pacientes que necessitaram de reperfusão mecânica após a TF. ${ }^{(14,17)}$ A ICP após TF por falha desta terapêutica foi necessária em $40 \%$ dos pacientes diabéticos, duas vezes mais do que nos pacientes não-diabéticos, $o$ que pode indicar uma menor eficácia da TF nestes pacientes. Isso é justificado pelo fato de apresentarem aumento da atividade plaquetária e níveis elevados de procoagulantes, e consequentemente um processo de fibrinólise comprometido. ${ }^{(16)}$

$\mathrm{O}$ encaminhamento precoce para a angiografia com ICP deve ser o padrão de atendimento após TF na chamada estratégia "farmacoinvasiva", quando necessário. Assim, a TF com posterior encaminhamento para um centro capaz de realizar a ICP pode alcançar resultados semelhantes ou superiores à ICPP. ${ }^{(27)}$

\section{Desfechos primários}

Para este estudo foram considerados como desfechos primários o óbito, o reinfarto e o Acidente Vascular Encefálico. Diversos ECR apresentaram segurança na administração pré-hospitalar da TF com otimização do tempo para início da terapia de reperfusão. ${ }^{(14,15,18,21,31,32)}$ Uma metanálise com ECR relevou que a administração pré-hospitalar diminui em até $17 \%$ o risco de morte quando comparada com a TF hospitalar. ${ }^{(33)}$ Em um estudo de metanálise realizado com 23 ECR foi encontrado que os pacientes submetidos a ICPP apresentaram taxas mais baixas de reinfarto não fatal e AVE a curto prazo quando comparados a pacientes submetidos a TF. ${ }^{(34)} \mathrm{O}$ AVE é uma das complicações que pode acontecer em decorrência do uso da TF. Os preditores independentes para a ocorrência do AVE quando administrada a TF sáo a idade avançada, o baixo peso, sexo feminino, antecedente de doença cerebrovascular e hipertensão arterial tanto sistólica como diastólica na admissão. ${ }^{(35,36)} \mathrm{O}$ tempo entre o início dos sintomas e a intervenção para reperfusão miocárdica também influenciou na mortalidade, mas não na ocorrência de reinfarto não-fatal e AVE. Foi observado maior risco de reinfarto para os pacientes diabéticos devido ao processo fisiopatológico coronariano. ${ }^{(16)}$ 


\section{Eventos adversos após 0 uso da terapia de reperfusão}

Em relação a TF pré-hospitalar existe um risco menor de choque cardiogênico, entretanto, está associado a um aumento do risco para Hemorragia Intracerebral (HI). Esse risco aumentado de HI reduziu pela metade a dose do tenecteplase nos pacientes com mais de 75 anos, o que diminuiu significativamente a taxa de sangramento no grupo TF e levou a taxas semelhantes entre os grupos. (14) Foi constatado um menor número de complicaçôes hemorrágicas náo cerebrais e menor transfusão sanguínea com o uso do tenecteplase, que possui maior afinidade pela fibrina e com isso menor risco de hemorragias. ${ }^{(24,27)} \mathrm{O}$ choque cardiogênico se apresenta como complicaçáo ocasionada pelo próprio IAMCST em quadros de isquemia extensa ou por complicaçôes mecânicas e geralmente ocorre nas primeiras 24 horas. ${ }^{(27)}$ Observa-se menor número de choque cardiogênico na população submetida à TF pré-hospitalar e o tempo constituiu fator protetor para a ocorrência deste quadro no grupo $\mathrm{TF}^{(14-16)}$

\section{Conclusão}

A administração da TF pré-hospitalar para pacientes com IAMCST mostrou-se oportuna ou até superior à ICPP com resultados semelhantes em benefícios, principalmente relacionados a mortalidade. Nos pacientes que receberam qualquer terapia de reperfusão até duas horas após o início dos sintomas, a TF pré-hospitalar imediata com encaminhamento para centro capaz de realizar ICP pode ser ainda mais vantajosa. $\mathrm{O}$ reinfarto não-fatal e a $\mathrm{HI}$ foram mais frequentes quando realizada a TF, entretanto, o choque cardiogênico ocorreu em menor número. Pacientes com diabetes podem ser beneficiados com a ICPP. A TF pré-hospitalar consiste numa oportunidade de tratamento viável para regiốes onde a ICPP não pode ser alcançada em tempo hábil. É importante que novos estudos avaliem e comparem o uso destas terapias para melhor compreensão e mais pacientes possam ser beneficiados com o seu uso adequado e oportuno.

\section{Agradecimentos}

Esse estudo contou com o apoio financeiro da Universidade Federal de Mato Grosso do Sul (UFMS) e com apoio da Coordenação de Aperfeiçoamento de Pessoal de Nível Superior Brasil (CAPES) - Código de Financiamento 001.

\section{Referências}

1. Thomas H, Diamond J, Vieco A, Chaudhuri S, Shinnar E, Cromer S, et al. Global Atlas of Cardiovascular Disease 2000-2016: the path to prevention and control. Glob Heart. 2018;13(3):143-63.

2. Cascaldi BG, Lacerda FM, Rodrigues A, Arruda GV. Infarto agudo do miocárdio sob a ótica da população brasileira. Rev Bras Cardiol. 2014;27(6):409-17.

3. Schmidt MM, Quadros AS, Martinelli ES, Gottschall CA. Prevalência, etiologia e características dos pacientes com infarto agudo do miocárdio tipo 2. Rev Bras Cardiol Invasiva. 2015;23(2):119-23.

4. O'Gara PT, Kushner FG, Ascheim DD, Casey DE Jr, Chung MK, de Lemos JA, et al. ACCF/AHA Guideline for the Management of ST-Elevation Myocardial Infarction. J Am Coll Cardiol. 2013;61(4):78-140.

5. Jneid H, Addison D, Bhatt DL, Fonarow GC, Gokak S, Grady KL, et al. 2017 AHA/ACC Clinical Performance and Quality Measures for Adults With ST-Elevation and Non-ST-Elevation Myocardial Infarction: A Report of the American College of Cardiology/American Heart Association Task Force on Performance Measures. J Am Coll Cardiol. 2017;70(16):2048-90.

6. O'Connor RE, Al Ali AS, Brady WJ, Ghaemmaghami CA, Menon V, Welsford M, et al. Part 9: Acute Coronary Syndromes: 2015 American Heart Association Guidelines Update for Cardiopulmonary Resuscitation and Emergency Cardiovascular Care. Circulation. 2015;132(18 Suppl 2):S483-500.

7. Levine GN, Bates ER, Blankenship JC, Bailey SR, Bittl JA, Cercek B, et al. 2011 ACCF/AHA/SCAI Guideline for Percutaneous Coronary Intervention: executive summary: a report of the American College of Cardiology Foundation/American Heart Association Task Force on Practice Guidelines and the Society for Cardiovascular Angiography and Interventions. Circulation. 2011;124(23):2574-609.

8. Mullasari A. Strategy of in ambulance thrombolysis followed by routine PCl in acute myocardial infarction. Indian Heart J. 2009;61(5):448-53.

9. Moeini M, Mahmoudian SN, Khalifezadeh A, Pour AH. Reviewing time intervals from onset of the symptoms to thrombolytic therapy in patients with ST segment elevation myocardial infarction (STEMI). Iran J Nurs Midwifery Res. 2010;15 Suppl 1:379-85.

10. Widimsky P, Wijns W, Fajadet J, de Belder M, Knot J, Aaberge L, et al.; European Association for Percutaneous Cardiovascular Interventions. Reperfusion therapy for ST elevation acute myocardial infarction in Europe: description of the current situation in 30 countries. Eur Heart J. 2010;31(8):943-57.

11. Pinto DS, Kirtane AJ, Nallamothu BK, Murphy SA, Cohen DJ, Laham RJ, et al. Hospital delays in reperfusion for ST-elevation myocardial infarction: implications when selecting a reperfusion strategy. Circulation. 2006;114(19):2019-25. 
12. Moher D, Liberati A, Tetzlaff J, Altman DG; PRISMA Group. Preferred reporting items for systematic reviews and meta-analyses: the PRISMA statement. Ann Intern Med. 2009;151(4):264-9.

13. Brasil. Ministério da Saúde. Secretaria de Ciência, Tecnologia e Insumos Estratégicos. Departamento de Ciência e Tecnologia. Diretrizes metodológicas: elaboração de revisão sistemática e metanálise de ensaios clínicos randomizados. Brasília (DF): Ministério da Saúde; 2012.

14. Armstrong PW, Gershlick AH, Goldstein P, Wilcox R, Danays T, Lambert $Y$, et al.; STREAM Investigative Team. Fibrinolysis or primary $\mathrm{PCl}$ in ST-segment elevation myocardial infarction. N Engl J Med. 2013;368(15):1379-87.

15. Bonnefoy E, Lapostolle F, Leizorovicz A, Steg G, McFadden EP, Dubien PY, et al.; Comparison of Angioplasty and Prehospital Thromboysis in Acute Myocardial Infarction study group. Primary angioplasty versus prehospital fibrinolysis in acute myocardial infarction: a randomised study. Lancet. 2002;360(9336):825-9.

16. Bonnefoy E, Steg PG, Chabaud S, Dubien PY, Lapostolle F, Boudet F, et al. Is primary angioplasty more effective than prehospital fibrinolysis in diabetics with acute myocardial infarction? Data from the CAPTIM randomized clinical trial. Eur Heart J. 2005;26(17):1712-8.

17. Bonnefoy E, Steg PG, Boutitie F, Dubien PY, Lapostolle F, Roncalli J, et al.; CAPTIM Investigators. Comparison of primary angioplasty and pre-hospital fibrinolysis in acute myocardial infarction (CAPTIM) trial: a 5-year follow-up. Eur Heart J. 2009;30(13):1598-606.

18. Steg PG, Bonnefoy E, Chabaud S, Lapostolle F, Dubien PY, Cristofini P, et al.; Comparison of Angioplasty and Prehospital Thrombolysis In acute Myocardial infarction (CAPTIM) Investigators. Impact of time to treatment on mortality after prehospital fibrinolysis or primary angioplasty: data from the CAPTIM randomized clinical trial. Circulation. 2003;108(23):2851-6.

19. Armstrong PW, Boden WE. Reperfusion paradox in ST-segment elevation myocardial infarction. Ann Intern Med. 2011;155(6):389-91.

20. Reddy K, Khaliq A, Henning RJ. Recent advances in the diagnosis and treatment of acute myocardial infarction. World $\mathrm{J}$ Cardiol. 2015;7(5):243-76.

21. Morrow DA, Antman EM, Sayah A, Schuhwerk KC, Giugliano RP, deLemos JA, et al. Evaluation of the time saved by prehospital initiation of reteplase for ST-elevation myocardial infarction: results of The Early Retavase-Thrombolysis in Myocardial Infarction (ER-TIMI) 19 trial. J Am Coll Cardiol. 2002;40(1):71-7.

22. Thygesen K, Alpert JS, Jaffe AS, Chaitman BR, Bax JJ, Morrow DA, et al. Fourth Universal Definition of Myocardial Infarction (2018). Eur Heart J. 2019;40(3):237-69.

23. Rawles J; GREAT. GREAT: 10 year survival of patients with suspected acute myocardial infarction in a randomised comparison of prehospital and hospital thrombolysis. Heart. 2003;89(5):563-4.

24. Dalal J, Sahoo PK, Singh RK, Dhall A, Kapoor R, Krishnamurthy A, et al. Role of thrombolysis in reperfusion therapy for management of AMl: indian scenario. Indian Heart J. 2013;65(5):566-85.
25. Ali MR, Salim Hossain M, Islam MA, Saiful Islam Arman M, Sarwar Raju G, Dasgupta P, et al. Aspect of thrombolytic therapy: a review. ScientificWorldJournal. 2014;2014:586510.

26. Nordt TK, Bode C. Thrombolysis: newer thrombolytic agents and their role in clinical medicine. Heart. 2003;89(11):1358-62.

27. Task Force on the management of ST-segment elevation acute myocardial infarction of the European Society of Cardiology (ESC), Steg PG, James SK, Atar D, Badano LP, Blömstrom-Lundqvist C, Borger MA, Di Mario C, Dickstein K, Ducrocq G, Fernandez-Aviles F, Gershlick AH, Giannuzzi P, Halvorsen S, Huber K, Juni P, Kastrati A, Knuuti J, Lenzen MJ, Mahaffey KW, Valgimigli M, van 't Hof A, Widimsky P, Zahger D. ESC Guidelines for the management of acute myocardial infarction in patients presenting with ST-segment elevation. Eur Heart J. 2012;33(20):2569-619.

28. Di Mario C, Dudek D, Piscione F, Mielecki W, Savonitto S, Murena E, et al.; CARESS-in-AMl (Combined Abciximab RE-teplase Stent Study in Acute Myocardial Infarction) Investigators. Immediate angioplasty versus standard therapy with rescue angioplasty after thrombolysis in the Combined Abciximab REteplase Stent Study in Acute Myocardial Infarction (CARESS-in-AMI): an open, prospective, randomised, multicentre trial. Lancet. 2008;371(9612):559-68.

29. Bøhmer E, Hoffmann P, Abdelnoor M, Arnesen H, Halvorsen S. Efficacy and safety of immediate angioplasty versus ischemia-guided management after thrombolysis in acute myocardial infarction in areas with very long transfer distances results of the NORDISTEMI (NORwegian study on Dlstrict treatment of ST-elevation myocardial infarction). J Am Coll Cardiol. 2010;55(2):102-10.

30. Cantor WJ, Fitchett D, Borgundvaag B, Ducas J, Heffernan M, Cohen EA, et al.; TRANSFER-AMI Trial Investigators. Routine early angioplasty after fibrinolysis for acute myocardial infarction. $\mathrm{N}$ Engl $\mathrm{J}$ Med. 2009;360(26):2705-18.

31. GREAT Group. Feasibility, safety, and efficacy of domiciliary thrombolysis by general practitioners: grampian region early anistreplase trial. BMJ. 1992;305(6853):548-53.

32. Pedley DK, Bissett K, Connolly EM, Goodman CG, Golding I, Pringle $\mathrm{TH}$, et al. Prospective observational cohort study of time saved by prehospital thrombolysis for ST elevation myocardial infarction delivered by paramedics. BMJ. 2003;327(7405):22-6.

33. Morrison LJ, Verbeek PR, McDonald AC, Sawadsky BV, Cook DJ. Mortality and prehospital thrombolysis for acute myocardial infarction: A meta-analysis. JAMA. 2000;283(20):2686-92.

34. Keeley EC, Boura JA, Grines CL. Primary angioplasty versus intravenous thrombolytic therapy for acute myocardial infarction: a quantitative review of 23 randomised trials. Lancet. 2003;361(9351):13-20.

35. Piegas LS, Timerman A, Feitosa GS, Nicolau JC, Mattos LA, Andrade MD, et al. V Diretriz Da Sociedade Brasileira de Cardiologia Sobre Tratamento do Infarto Agudo do Miocárdio com Supradesnível do Segmento ST. Arq Bras Cardiol. 2015;105(2):1-105.

36. Armstrong PW, Gershlick A, Goldstein P, Wilcox R, Danays T, Bluhmki E, et al.; STREAM Steering Committee. The Strategic Reperfusion Early After Myocardial Infarction (STREAM) study. Am Heart J. 2010;160(1):30-35.e1. 